\title{
Indicadores de agregación reproductiva de Thais chocolata (Duclos, 1832) (Gastropoda, Thaididae) en Caleta Punta Arenas $\left(21^{\circ} 38 ' S-70^{\circ} 09^{\prime} \mathrm{W}\right)^{*}$
}

\author{
Miguel Avendaño ${ }^{1}$, Marcela Cantillánez ${ }^{1}$, Alberto Olivares $^{1}$ y Marcelo Oliva ${ }^{2}$ \\ ${ }^{1}$ Departamento de Acuicultura \\ ${ }^{2}$ Instituto de Investigaciones Oceanológicas \\ Universidad de Antofagasta, Casilla 170, Antofagasta, Chile. \\ E-mail: mavendano@uantof.cl
}

\begin{abstract}
RESUMEN. El análisis de la densidad, distribución espacial e histología de gónadas de Thais chocolata en caleta Punta Arenas $\left(21^{\circ} 38^{\prime} \mathrm{S}-70^{\circ} 09^{\prime} \mathrm{W}\right)$, permitió definir ciertos indicadores que señalan que las agregaciones reproductivas se constituirían en la zona somera (13-15 m de profundidad), en las mismas épocas que se han señalado para otras poblaciones de esta especie de la zona norte de Chile. Los indicadores que evidencian este acontecimiento se refieren a la distribución agregada de organismos adultos sexualmente maduros en los veriles de los $13 \mathrm{~m}$ y $15 \mathrm{~m}$, en diferentes épocas del año. La constitución de estas agregaciones en períodos diferentes al de la veda establecida para este recurso obliga el reestudio de esta norma, con el objeto de proteger su reproducción e impedir un eventual agotamiento del stock en las Regiones I y II $\left(18^{\circ}-26^{\circ} \mathrm{S}\right)$, considerando que la pesquería que existía en las Regiones III y IV $\left(26^{\circ}-32^{\circ} \mathrm{S}\right)$ colapsó en 1988.
\end{abstract}

Palabras claves: Thais chocolata, locate, gastrópodo, agregación reproductiva, Chile.

\section{Indicators of the reproductive aggregations of Thais chocolata (Duclos, 1832) (Gastropoda, Thaididae) in Caleta Punta Arenas $\left(2^{\circ}{ }^{\circ} 38^{\prime} \mathrm{S}-\mathbf{7 0}^{\circ} 09^{\prime} \mathrm{W}\right)^{*}$}

\begin{abstract}
The analysis of density, spatial distribution and gonadic histology of a Thais chocolata population in small bay Punta Arenas $\left(21^{\circ} 38^{\prime} \mathrm{S}-70^{\circ} 09^{\prime} \mathrm{W}\right)$, has allowed define certain indicators, which show that the reproductive aggregations would take place in the shallow zone (13-15 m depth) at the same time reported for other populations of this species in northern Chile. The feature that evidence such an event are referred to the aggregated distribution of sexually mature adults in the $13 \mathrm{~m}$ and $15 \mathrm{~m}$ isobath, a distinct times of the year. Such times are different from these established for the legal veda. Thus, this legal reduction should be restudied in order to protect reproduction of this specie and hence to prevent a potential collapse of the stock in the Regions I and II $\left(18^{\circ}-26^{\circ} \mathrm{S}\right)$, taking into account that the previous fishery in Regions III and IV $\left(26^{\circ}-32^{\circ} \mathrm{S}\right)$ have already collapsed since 1988.
\end{abstract}

Key words: Thais chocolata, "locate", gastropod, reproductive aggregation, Chile.

\section{INTRODUCCION}

El caracol de nombre común "locate" (Thais chocolata Duclos, 1832), se distribuye desde el límite norte del país hasta Valparaíso en Chile (Osorio, 1979), es una especie gonocórica con fertilización interna, sin evidencias externas de dimorfismo sexual (Rojas et al., 1986). Durante la época de reproducción los adultos se concentran formando agrupaciones conocidas como "maicillo" (Retamales y González, 1982), tal como ocurre con Thais lamellosa (Bertness, 1977). Esta conducta ha sido comunicada en Chile para Concholepas concholepas (Ramorino, 1975; Castilla, 1979), y también para gastrópodos caribeños tales como Strombus gigas, que se reproduce durante todo el año realizando la cópula en aguas someras (Berg y Olsen, 1989).

El locate constituyó un recurso bentónico de importancia económica para la pesquería artesanal del norte de Chile (Retamales y González, 1982), concentrándose en aguas someras. Sin embargo, debi-

* Trabajo desarrollado a través del Proyecto Cod. BIP N 20052251-2. 
do a los altos niveles de explotación que se ejercieron desde los inicios de su pesquería en 1978, y después de haberse alcanzado el máximo desembarque en 1986 con 8.244 ton, la fracción vulnerable de la zona somera se redujo fuertemente. Así, el área de distribución de la pesquería se restringió a las Regiones I y II $\left(18^{\circ}-26^{\circ} \mathrm{S}\right)$, ya que a partir de 1988 la abundancia del recurso en las Regiones III y IV $\left(26^{\circ}\right.$. $32^{\circ} \mathrm{S}$ ) no sustentó niveles de captura comerciales (SUBPESCA, 1995). En 1985 se estableció la talla mínima legal en $55 \mathrm{~mm}$, medida a lo largo de su eje máximo (Decreto 427 del 6 de diciembre de 1985) y una veda biológica entre el 1 de enero y el 31 de marzo de cada año. Pese a estas medidas de protección, los niveles de desembarque siguen disminuyendo, registrándose en 1996 tan sólo 1.568 ton (SUBPESCA, 1997).

Situaciones similares de sobrepesca han sido registradas para otras especies. Berg y Olsen (1989) señalan que la distribución y abundancia del gastrópodo Strombus gigas han sido gravemente afectadas por la presión de pesca, lo que también ha sido demostrado por Iversen y Jory (1997) para esta misma especie. Moreno y Reyes (1988) indican que la captura sostenida de individuos de tallas mayores de Concholepas concholepas han afectado la cantidad de individuos reproductivos, generando una reducción de las capturas de 4 mil ton en 1984 a menos de 1 ton en 1988 para la zona de Valdivia $\left(40^{\circ} \mathrm{S}\right)$.

La alta actividad extractiva observada sobre Thais chocolata, en especial aquella asociada con las agrupaciones reproductivas (conducta gregaria) que ocurren en aguas someras durante los períodos reproductivos, ha sido poco estudiada en el país. En el presente trabajo se describen los cambios de densidad poblacional, distribución de tallas por profundidad y grado de madurez de hembras en aguas someras, como indicadores de períodos reproductivos que acontecen anualmente, los cuales pueden contribuir a la revisión de la veda que rige actualmente para esta especie.

\section{MATERIALES Y METODOS}

El estudio se desarrolló en caleta Punta Arenas (Fig. 1), entre mayo de 1995 y mayo de 1996. El área de distribución local de Thais chocolata se determinó mediante buceo. El criterio de delimitación consistió en reconocer primero la presencia de esta especie dentro de la zona seleccionada; luego se definieron tres transectas perpendiculares a la costa, separadas entre sí cada $100 \mathrm{~m}$. Para caracterizar la existencia de variaciones temporales, en cada una de ellas se determinó mensualmente la densidad de individuos presentes por veril de profundidad, muestreando siempre desde la mayor profundidad.

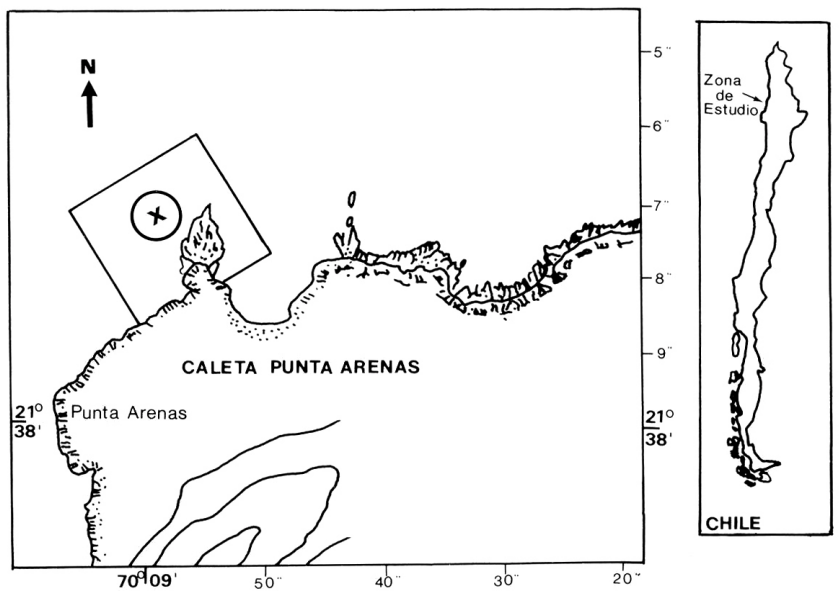

Figura 1. Ubicación geográfica de caleta Punta Arenas y del área de estudio (X).

Figure 1. Geographic location of Caleta Punta Arenas and study area (X). 
Para ello se dejó caer al azar un cuadrante de $1 \mathrm{~m}^{2}$, el cual fue replicado 4 veces cada $5 \mathrm{~m}$ de profundidad. La definición de las tres transectas y la restricción del muestreo a sólo $4 \mathrm{~m}^{2}$ por veril de profundidad, se debió a razones propias de la limitación del tiempo de buceo.

Adicionalmente al muestreo sistemático, una vez concluido el estudio, se realizaron dos observaciones de agregación reproductiva con cópula y postura de cápsulas con huevos. En junio de 1996 se detectaron seis agregaciones, contabilizándose entre 67 y 312 individuos por agregación, mientras que en octubre de 1996 se observaron cuatro agregaciones, compuestas de 42 a 256 ejemplares.

La caracterización del patrón de distribución del locate se realizó aplicando el índice estandarizado de dispersión de Morisita, el cual no es afectado por la densidad ni por el tamaño de la muestra. Este índice fluctúa entre -1 y +1 , con límites de confianza (95\%) entre $-0,5$ y +0,5 (Krebs, 1989).

Para conocer la estructura de talla por veril de profundidad, los ejemplares presentes en cada cuadrante analizado fueron recogidos, subidos a bordo de la embarcación y medidos en su longitud total (en su eje máximo) con un pie de metro de $0,1 \mathrm{~mm}$ de precisión. Posteriormente, las partes blandas fueron separadas de la concha rompiéndola cuidadosamente. El estado de madurez sexual se caracterizó de acuerdo a los criterios indicados por Retamales y González (1982). Todos los ejemplares sexados inequívocamente como hembras fueron utilizados para el análisis histológico. El número de hembras por muestra y veril de profundidad, fluctuó entre 15 y 30. Se fijaron secciones de la zona media del conjunto glándula digestiva-gónada en Bouin alcohólico. El análisis histológico del ovario se realizó mediante la técnica tradicional, con inclusión en parafina, obteniéndose cortes de $6 \mathrm{~mm}$ que fueron teñidos con hematoxilina-eosina.

\section{RESULTADOS}

El área de estudio se caracterizó por presentar fondos duros de baja profundidad ("bajos rocosos") cubiertos por cholgas (Aulacomya ater), sobre las cuales se encuentra Thais chocolata. Los bajos se extienden entre 13 y $25 \mathrm{~m}$ de profundidad, separados por "canalones" de arena gruesa.

Durante el muestreo, Thais chocolata se encontró habitualmente distribuido entre los 25 y $15 \mathrm{~m}$ de profundidad, aunque en junio de 1995 y marzo de 1996 se encontró a los 13 m, con densidades promedios de 4 ind $* m^{-2}$ y 3 ind $* m^{-2}$, respectivamente (Fig. 2). No obstante, y pese a que durante estos meses no se detectó la formación de agregaciones reproductivas, debido a la fuerte presión extractiva observada, se encontró esporádicamente posturas de cápsulas con huevos en el veril de $13 \mathrm{~m}$ de profundidad.

El análisis de los patrones de distribución de los organismos mediante el índice de Morisita (Tabla 1), mostró que durante la época reproductiva, los locates se agrupan preferentemente en los veriles menos profundos.

La talla de los organismos examinados varió

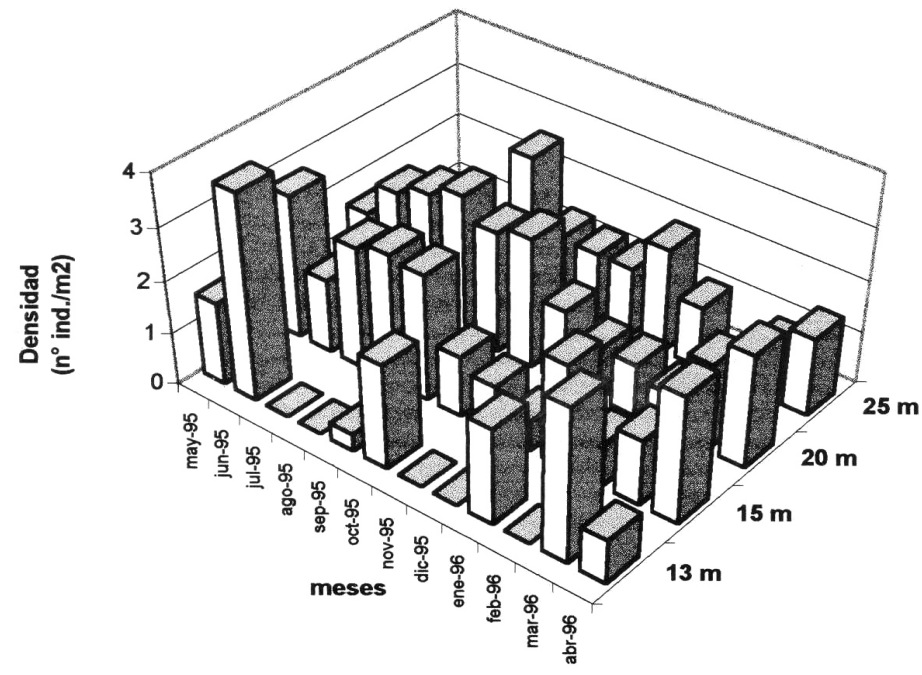

Figura 2. Densidad promedio mensual de locates por veril de profundidad en caleta Punta Arenas.

Figure 2. Mean densities monthly of locate, by isobath in Caleta Punta Arenas. 
Tabla I. Valores del índice estandarizado de Morisita, para las distintas profundidades en Caleta Punta Arenas. Valores resaltados indican distribución agregada.

Table I. Standardized Morisita Index values for the differents isobath in Caleta Punta Arenas. Remarked values show aggregated distributions.

\begin{tabular}{|c|c|c|c|c|}
\hline & \multicolumn{3}{|c|}{ Profundidad } & \\
\hline Meses & $25 \mathrm{~m}$ & $20 \mathrm{~m}$ & $15 \mathrm{~m}$ & $13 \mathrm{~m}$ \\
\hline may-95 & & 1,26 & 2,05 & 0,98 \\
\hline jun-95 & & 1,13 & 1,85 & 1,21 \\
\hline jul-95 & & $\overline{1,46}$ & 2,06 & \\
\hline ago-95 & 1,45 & $\overline{1,14}$ & $\overline{1}, 10$ & \\
\hline sep-95 & 1,59 & 1,21 & 1,15 & 12,00 \\
\hline oct-95 & 1,40 & 1,43 & 2,24 & 1,35 \\
\hline nov-95 & 0,80 & 0,88 & 1,53 & \\
\hline dic-95 & 1,35 & 0,55 & 0,57 & \\
\hline ene-96 & 1,19 & 0,91 & 1,78 & 3,01 \\
\hline feb-96 & & 1,00 & 0,80 & \\
\hline mar-96 & 0,91 & 1,07 & 1,26 & 1,11 \\
\hline abr-96 & 1,33 & 2,10 & 1,36 & 3,73 \\
\hline
\end{tabular}

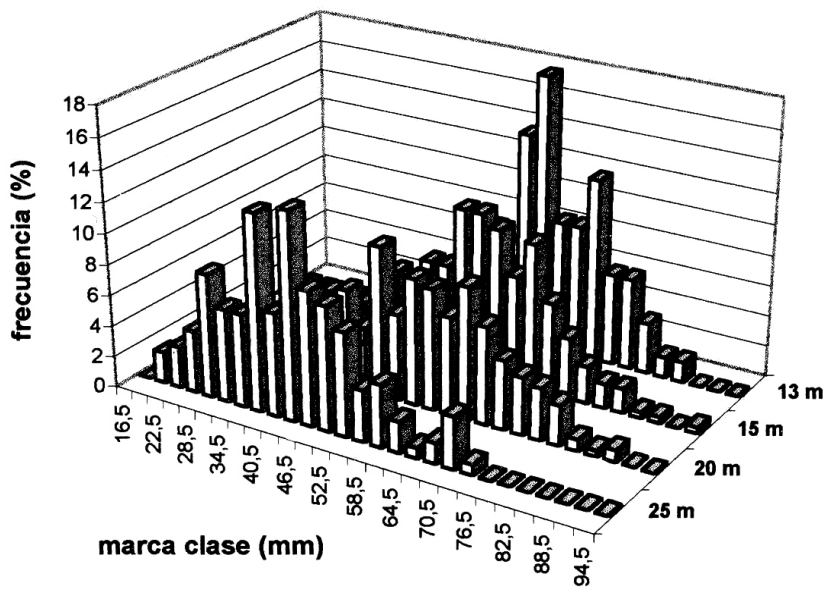

Figura 3. Estructura de tallas de locates por veril de profundidad durante el período de estudio en caleta Punta Arenas.

Figure 3. Size structure of locate by isobath for the total period of study in Caleta Punta Arenas. entre 15 y $96 \mathrm{~mm}$ de longitud. El $84 \%$ de los especímenes obtenidos a los $13 \mathrm{~m}$, el $64 \%$ a los $15 \mathrm{~m}$ y el $53 \%$ a los $20 \mathrm{~m}$ correspondieron a individuos mayores de $54 \mathrm{~mm}$. A su vez, a los $25 \mathrm{~m}$ de profundidad predominaron las tallas inferiores a $54 \mathrm{~mm}$ (Fig. 3), que representaron el 58,7\% del total de individuos examinados. Durante los meses en que se observó la presencia de Thais chocolata a $13 \mathrm{~m}$ (Fig. 2), el porcentaje de individuos mayores de $54 \mathrm{~mm}$ varió entre $79 \%$ y $89 \%$.

Los resultados histológicos del ovario indicaron que los estados maduros se encontraron frecuentemente en los veriles menos profundos. Debido a esto, se graficaron los porcentajes mensuales de hembras maduras presentes en los veriles de 15 y $13 \mathrm{~m}$ de profundidad, que variaron entre 15 y $100 \%$ (Fig. 4). Se observaron incrementos de este porcentaje en mayo, julio, septiembre y diciembre de 1995 , y marzo y mayo de 1996, en correspondencia simultánea o previa a la presencia de los organismos en el veril de $13 \mathrm{~m}$.

\section{DISCUSION}

La ocurrencia de agregaciones reproductivas de Thais chocolata se observaron sólo en junio y octubre de 1996; mientras que Avendaño et al. (1997), en un estudio paralelo desarrollado en el sector de La Rinconada $\left(23^{\circ} 40^{\prime} \mathrm{S}\right)$, encontraron agregaciones en aguas someras en diferentes períodos del año. Sin embargo, siendo habitual encontrar a los ejemplares distribuidos entre los 25 $\mathrm{m}$ y $15 \mathrm{~m}$ de profundidad, se pudo constatar en mayo, junio, septiembre y octubre de 1995, y enero, marzo y abril de 1996, la presencia de ejemplares a los $13 \mathrm{~m}$, indicando que las agregaciones acontecen en los mismos períodos en que se registraron en La Rinconada. La extensión 


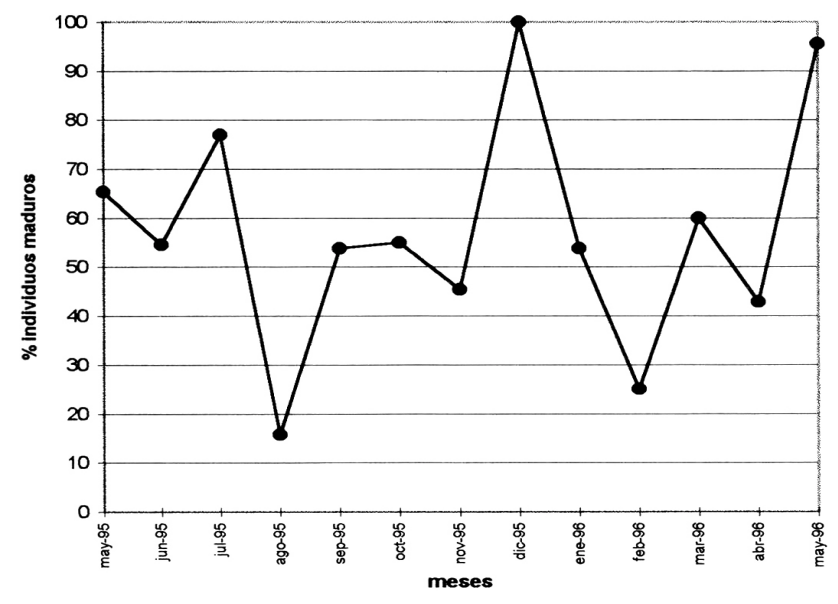

Figura 4. Porcentaje mensual de hembras maduras de locates en caleta Punta Arenas.

Figure 4. Monthly percentage of mature locate females, in Caleta Punta Arenas.

en la distribución del locate hasta esta profundidad, está caracterizada además, por un aumento en la densidad de organismos, constituyendo agrupaciones que fueron confirmadas mediante el índice de Morisita.

Otro hecho importante que confirma que las agregaciones reproductivas ocurren durante esos meses, se observó mediante el análisis histológico de las gónadas, que indicó que las hembras encontradas a los $13 \mathrm{~m}$ eran predominantemente maduras, alcanzando en septiembre de 1995, y mayo de 1996 el $100 \%$ y el $97 \%$ respectivamente. Por otro lado, el análisis global de los porcentajes de hembras maduras, en los veriles de 13 y $15 \mathrm{~m}$ de profundidad, mostró valores elevados en mayo, julio y septiembre de 1995, y en marzo y mayo de 1996, coincidiendo con los resultados obtenidos para $\mathrm{La}$ Rinconada (Avendaño et al., 1997), y con los obtenidos por Retamales y González (1982) para la Península Serrano $\left(20^{\circ} 12^{\prime} \mathrm{S}\right)$, lo que indicaría que los procesos reproductivos ocurren simultáneamente en las poblaciones de Thais chocolata localizadas entre la I y II Regiones $\left(18^{\circ}-26^{\circ} \mathrm{S}\right)$.

La estructura de talla de los organismos analizados a los diferentes niveles de profundidad, muestra que aquéllos de tallas inferiores a la mínima le- gal (55 $\mathrm{mm}$ de longitud total), generalmente se distribuyen a mayor profundidad. Así, cerca del $60 \%$ de los individuos de 15 a $54 \mathrm{~mm}$ se localizan a los $25 \mathrm{~m}$, mientras que las tallas mayores de $54 \mathrm{~mm}$ se distribuyen en veriles menos profundos, sobrepasando el $80 \%$ de los locates de esta población. Esta estructura de talla predominó en aquellos organismos maduros que se distribuyeron en determinados meses, hasta el veril de $13 \mathrm{~m}$

La detección de agregaciones reproductivas sólo en junio y octubre de 1996, a diferencia de lo señalado por Avendaño et al. (1997) para La Rinconada, muestra la vulnerabilidad del locate a su total extracción por parte de buzos mariscadores a través de todo el año, así también, como a la de organismos de tallas mayores que se acercan agrupados a reproducirse a estos someros veriles de profundidad. Esto evidencia que la veda biológica para este recurso, expresada en el Decreto Supremo N ${ }^{\circ} 427$ de 1985, no es coincidente con su período reproductivo.

La interrupción de este importante proceso biológico pone en grave riesgo la conservación del recurso, situación que en el corto plazo puede llevar a su agotamiento y eventualmente, al colapso de la pesquería de Thais chocolata, por la fuerte reducción del stock debido a los altos niveles de explotación ejercidos desde 1986 a la fecha, que han sobrepasado los niveles técnicamente recomendados para su adecuada conservación (SUBPESCA, 1995).

Además, de acuerdo a Rivas y Canales (1994), se puede señalar que los desembarques de este recurso, se sustentan principalmente por la explotación de una fracción no despreciable de su stock, en vez de sus excedentes productivos. Esto es análogo a lo señalado por Moreno y Reyes (1988), para Concholepas concholepas en la región de Valdivia, donde las capturas sostenidas de individuos de tallas mayores, han afectado la cantidad de organismos que participan en la reproducción, la abundancia de larvas en el plancton, el número de individuos asentados al bentos y los reclutas a la población intermareal, reduciéndose considerablemente su captura. 
Finalmente, información histórica obtenida en Hornitos por Miranda (1967), señala que las agregaciones de locates que ocurren actualmente en la zona submareal, hace unas décadas atrás ocurrían en los roqueríos de la zona intermareal, tal como aconteció con T. lamellosa (Bertness, 1977). Esto lleva a concluir la necesidad de actualizar los antecedentes en que se basa la veda biológica aplicada a este recurso bentónico.

\section{REFERENCIAS}

\section{Avendaño, M., M. Cantillánez, A. Olivares y M. Oli-} va. 1997. Conducta reproductiva de Thais chocolata (Duclos, 1832) (Gastropoda:Thaididae), en La Rinconada, Antofagasta-Chile: Causal de vulnerabilidad a la pesca. Rev. Biol. Mar. Oceanogr., 32(2): 177-187.

Berg, C.J. y D.A. Olsen. 1989. Conservation and management of queen conch (Strombus gigas) fisheries in the Caribbean. In: J. F. Caddy (Ed.). Marine Invertebrate Fisheries: Their Asessment and Management. Publishes, Rome, pp. 421-442.

Bertness, M.D. 1977. Behavorial and ecological aspects of shore-level size gradients in Thais lamellosa and Thais emarginata. Ecology, 58 (1): 86-97.

Castilla, J.C. 1979. Concholepas concholepas (Mollusca, Gastropoda, Muricidae): Postura de cápsulas en el laboratorio y en la naturaleza. Biol. Pesq., Chile, 12: 91-97.

Iversen, E.S. y D.E. Jory. 1997. Mariculture and enhancement of wild population of queen conch (Strombus gigas) in the western Atlantica Full source. Bull. Mar. Sci., 60 (3): 929-941.

Krebs, C.J. 1989. Ecological methodology. Harper Collins Pub. New York, 654 pp.

Miranda, B.O. 1967. Edad y grupos modales de Thais chocolata: una descripción de los métodos usados. Apunt. Ocean., 3: 1-25.

Moreno, C. y A. Reyes. 1988. Densidad de Concholepas concholepas (Gastropoda, Muricidae) en la reserva marina de Mehuín: Evidencias de falla en el reclutamiento. Biol. Pesq., Chile, 17: 31-38.

Osorio, R.C. 1979. Moluscos marinos de importancia económica en Chile. Biol. Pesq., Chile, 11: 3-47.

Ramorino, L. 1975. Ciclo reproductivo de Concholepas concholepas en la zona de Valparaíso. Rev. Biol. Mar., 15(2): 149- 177.

Rivas, D. y C. Canales. 1994. Evaluación del stock del recurso locate (Thais chocolata) en las Regiones I y II. Informe Técnico Subsecretaría de Pesca, Chile. 10 pp.

Retamales, R. y L. González. 1982. Prospección, evaluación y reproducción del erizo, ostión y locate. Informe, SERPLAC-IFOP. 124 pp.

Rojas, N., J. Tarazona y V. Ishiyama. 1986. Ciclo de reproducción y escala de madurez gonadal en el "caracol" Thais (Stramonita) chocolata (Duclos, 1832). Rev. Cien. U.N.M.S.M., 74(1): 117-129 .

SUBPESCA. 1995. Regulación del acceso a la pesquería del caracol locate. Inf. Téc. Subsecretaría de Pesca, Chile, 16 pp. 